\title{
Potential role of therapeutic hypothermia in the salvage of traumatic hemorrhagic shock
}

Shou-yin Jiang, Ying-ying Zhao and Xiao-gang Zhao*

\begin{abstract}
Although therapeutic hypothermia could serve as a potential therapeutic strategy for treatment of traumatic hemorrhagic shock, significant controversy exists regarding its safety and feasibility. The current resuscitation strategy in traumatic hemorrhagic shock may also require updating. In this article, we have carried out an extensive literature search in this field and propose an initial algorithm for use of therapeutic hypothermia in traumatic hemorrhagic shock. This work lays essential groundwork for future investigations in this field.
\end{abstract}

\section{Introduction}

Hemorrhage remains the primary potentially preventable cause of death on battlefields and the second leading cause of death in civilian trauma [1,2]. Unfortunately, an estimated $56 \%$ of deaths occur during the pre-hospital period and $50 \%$ of patients died within 24 hours after admission as a result of hemorrhagic shock (HS) [3,4]. Among those found dead upon the arrival of emergency medical services personnel, exsanguination continues to be the most common cause of death [5]. Although $20 \%$ of deaths after HS are considered as avoidable [6], current practice does not improve survival rate significantly. Patients severely wounded in combat may still die even with adequate circulating blood volume and indicators of improving physiology [7].

Therapeutic hypothermia $(\mathrm{TH})$ has been introduced into the treatment of human disease. Up to now, it has been broadly used in cardiovascular surgery but has also been recommended for treatment of cardiac arrest, severe traumatic brain injury, near-drowning, ischemic stroke, neonatal hypoxic-ischemic encephalopathy, and spinal cord injury $[8,9]$. Recent studies have focused on

*Corresponding: zhaoxiaogang1969@gmail.com

Department of Emergency Medicine, Second Affiliated Hospital, Zhejiang University School of Medicine, 88 Jiefang Road, Hangzhou 310009, China its potential role in traumatic HS [10,11]. However, due to differences in methodology and animal models, the results remain inconclusive. Because of its various adverse effects, $\mathrm{TH}$ has not been attempted in clinical treatment of HS. In this article, we systematically review current studies regarding $\mathrm{TH}$ in $\mathrm{HS}$ and assess the potential of $\mathrm{TH}$ with regard to translation from basic research to clinical practice. Our review provides novel insights into the field and proposes a new initial hypothetical algorithm for use of TH in trauma and HS in the future. Considering the wide range of $\mathrm{TH}$ temperatures used in basic trauma studies, hypothermia in this paper is classified as described previously into five levels: mild (33 to $\left.36^{\circ} \mathrm{C}\right)$, moderate $\left(28\right.$ to $\left.32^{\circ} \mathrm{C}\right)$, deep $(16$ to $\left.27^{\circ} \mathrm{C}\right)$, profound $\left(6\right.$ to $\left.15^{\circ} \mathrm{C}\right)$, and ultra-profound $\left(<5^{\circ} \mathrm{C}\right)$ [11].

\section{Therapeutic hypothermia in (traumatic) hemorrhagic shock: a brief history of studies}

The use of TH in HS was first proposed in the early 1940s. Over the following decades, more studies regarding TH in HS have been published, with two-thirds published in the last 20 years. However, the first clinical trial in this field was not approved until 2011 [12]. Clinical translation has been impeded by safety considerations since laboratory findings were often inconsistent, even with the same methodology and depth of hypothermia. Meanwhile, the development of the trauma 'triad of death' [13] undoubtedly discouraged all previous studies. On the other hand, moderate and deep hypothermia $\left(<30^{\circ} \mathrm{C}\right)$ had been the primary interest in HS until the late 1990s, when mild hypothermia was introduced. It has been suggested that mild hypothermia is easier to implement and more feasible compared with moderate and deep hypothermia. Currently, mild and deep to profound hypothermia are mostly studied in the setting of HS. These studies are designed to explore whether TH would be a useful strategy to treat traumatic HS. Regardless of whether bleeding is controllable or uncontrollable after HS, basic studies have uncovered the crucial role of TH in improving survival outcome, apoptosis, oxidative stress and organ injury, organ performance, and visceral permeability [14]. A recent prominent 
meeting convened an expert panel to critically address the importance of TH in the rescue of traumatic HS [10]. In other cases, experts from different institutions have clarified their standpoints regarding the potential role of $\mathrm{TH}$ in trauma and HS [11,14-16]. Now two strategies have been proposed for the use of $\mathrm{TH}$ as suggested by laboratory studies and case reports: mild $\mathrm{TH}$ for the management of low flow status HS; and deep to profound $\mathrm{TH}$, namely emergency preservation and resuscitation (EPR) treatment, for the management of cardiac arrest following massive bleeding [17].

\section{Known biological mechanisms of therapeutic hypothermia}

Hypothermia induces various physiological changes in vivo. Early basic and clinical observational studies attributed beneficial effects of hypothermia exclusively to depression of metabolism. This concept was changed in the 1970s when mild hypothermia was introduced into laboratory studies. The kinetic properties of most enzyme systems are temperature-dependent; thus, various enzyme-mediated reactions are slowed down after cooling depending on its intensity. So it is not surprising to find that many in vivo and in vitro studies showed decreased levels of multiple molecules from different organs, such as nuclear factor kappa B (NF- $\mathrm{kB}$ ), inducible nitric oxide synthase (iNOS) [18], caspases [19], reactive oxygen species [20], hypoxia inducible factors (HIFs) [21], and pro-inflammatory cytokines [22]. Inhibition of such molecules is essential for protecting against cell damage. However, low body temperature does not inhibit all biochemical reactions. Molecules that are crucial for survival, such as Bcl-2 [19], heat shock protein and Akt [23], are found to be increased after hypothermia treatment during HS. Deep to profound TH has been shown to prevent cellular and/or tissue energy failure and thus the development of ischemia. This has become the core concept of EPR treatment for exsanguination cardiac arrest [10]. In terms of hemodynamics, mild $\mathrm{TH}$ can elevate blood pressure, mostly because of enhanced systemic vascular resistance and cardiac performance [24]. This mechanism, by supporting continuous blood flow, could be crucial for the survival benefits of TH in HS. As a result, TH may improve survival by suppressing death profiles and promoting survival profiles.

\section{New insights into hemorrhagic shock with therapeutic hypothermia treatment Therapeutic versus spontaneous hypothermia}

It has been shown that $\mathrm{TH}$ is different from spontaneous hypothermia [15]. In the initial period of traumatic HS, tissues lose their ability to maintain aerobic metabolism, converting to anaerobic metabolism after major blood loss. This change will take away large amounts of heat.
Tissue ischemia is followed by reduced production of ATP, leading to less production of heat. All these conditions lead to the development of initial hypothermia. Low body temperature will initially be compensated for by the shivering reaction but this disappears below $33.5^{\circ} \mathrm{C}$ [25]. However, shivering in HS does not produce significant amounts of heat but increases energy consumption. In addition, environmental and iatrogenic factors may enhance the cooling process, such as winter conditions, wet clothes, immobilization, anesthesia, infusion of cold fluids, and exposure to surgery [26]. Therefore, body temperature continues to decrease, acidosis and electrolyte disorders are aggravated and coagulopathy presents; these conditions consequently cause death [13]. Current TH practice, however, is based on preventing shivering so as to cool body temperature to 32 to $34^{\circ} \mathrm{C}$. Thus, the key difference between $\mathrm{TH}$ and spontaneous hypothermia is that $\mathrm{TH}$ is controlled and based on preventing shivering while spontaneous hypothermia is uncontrolled and involves shivering. This difference is important because some clinical studies have indicated that spontaneous hypothermia is deleterious and hypothermic trauma patients should be rewarmed to more than $35^{\circ} \mathrm{C}$ [25,27]. However, as mild hypothermia is increasingly accepted for its multiple beneficial effects, patients with moderate or deep spontaneous hypothermia after traumatic HS may benefit when they are rewarmed to mild hypothermia. One case report used this procedure in an accidental cardiac arrest combined with spontaneous deep hypothermia [28].

Nonetheless, TH and spontaneous hypothermia both involve physical cooling. Therefore, even spontaneous hypothermia could be beneficial (for example, for organ preservation). In most cases, however, only intervention will prevent deterioration of a patient with spontaneous hypothermia. The key issue is that the body temperature should be controlled. In a convincing study, Martin et al. [29] reported that although patients with admission hypothermia at $<32^{\circ} \mathrm{C}$ have high mortality, some do survive and mortality rates remain constant at temperatures between $27^{\circ} \mathrm{C}$ and $32^{\circ} \mathrm{C}$. What is the underlying mechanism for the reduced mortality rate? We believe this is a complicated process and organ preservation or lack of anti-shivering therapy may not be the only explanation. Likely, hypothermia per se may not be important because spontaneous hypothermia is usually determined by injury severity $[27,30]$.

\section{Therapeutic hypothermia and the trauma 'triad of death'}

It is essential to consider the effects of $\mathrm{TH}$ on the development of the trauma 'triad of death' - that is, acidosis, spontaneous hypothermia, and coagulopathy - in severely injuried patients during hypothermia treatment. These can develop quickly after trauma exsanguination and could 
form a vicious circle that may be impossible to overcome. However, TH may not lead to adverse effects on these factors. When traumatic HS patients are treated with $\mathrm{TH}$, body temperature would be controlled tightly without significant fluctuation. As a result, even when spontaneous hypothermia has already started, it will be effectively controlled under $\mathrm{TH}$. $\mathrm{TH}$ has been shown to prevent tissue ischemia because of energy preservation. Therefore, the metabolic acidosis would not be aggravated. With regard to the coagulation system, although the enzymatic activity of clotting factors and the function of platelets is reduced as temperature decreases, mild hypothermia alone in trauma patients may not significantly influence hemostasis [31,32]. While it is well recognized that coagulopathy occurs as the result of imbalances between pro-coagulation and anti-coagulation, the development of acute traumatic coagulopathy is also an inflammatory disorder. There is significant crosstalk between the coagulation and inflammation system [15]. In this regard, $\mathrm{TH}$ has been shown to modulate multiple inflammation reaction pathways [14]. Nonetheless, early and close monitoring of coagulation function and correct coagulopathy in accordance with damage control resuscitation are indispensable [33]. In patients with cardiac arrest caused by trauma exsanguination, the duration of initial deep to profound $\mathrm{TH}$ is largely dependent on surgery time, while its influence on coagulation, acid-base balance, and survival has yet to be identified. In any case, EPR treatment seems to be the primary choice for organ preservation in such circumstances.

\section{Step-by-step fluid resuscitation}

Limited fluid resuscitation (LFR) is routinely practiced in circumstances of uncontrolled traumatic HS. Is it reasonable to achieve early resuscitation endpoints after hemostasis and during hypothermia treatment? The current practice of resuscitation in HS suggests aggressive fluid resuscitation (AFR) after hemostasis, the goal of which is to achieve early restoration of a normal internal environment [34]. High morbidity and mortality may be related to failure to achieve resuscitation endpoints in the early phase [35]. However, early AFR is associated with increased cardiac dysfunction, abdominal compartment syndrome, acute respiratory distress syndrome, and multiple organ failure [36]. In contrast, mildly hypotensive resuscitation (MAP at $70 \mathrm{mmHg}$, as suggested in rats) after control of bleeding has been proposed by $\mathrm{Li}$ and colleagues [37]. The benefits of mildly hypotensive resuscitation can be attributed to alleviated oxidative stress injury or, broadly speaking, resuscitation injury [38]. A brief review of the studies performed by Douzinas and colleagues [39] reveals that resuscitation with low $\mathrm{FiO}_{2}$ (fraction of inspired oxygen) could protect ischemic tissue from reperfusion injury after HS. These studies indicate that restricting volume resuscitation may still be needed after hemostasis. It also seems reasonable to restrict fluid volume during hypothermia treatment since fluid overload may neutralize the protective effects of $\mathrm{TH}$.

In order to bridge the gap between LFR and AFR, we introduce the strategy of step-by-step fluid resuscitation (SSFR). This concept refers to resuscitating patients in a gradual fashion after control of bleeding until blood pressure reaches a certain threshold and is maintained for a certain period. The gradual fashion means resuscitating with fluids at a relatively lower rate, which makes it possible to increase blood pressure in a gentle way rather than a rapid elevation. It is also possible to maintain blood pressure at subnormal levels for a determined period so as to minimize ischemic-perfusion injury. However, optimization of the SSFR strategy is complicated because multiple parameters are variable, such as the ideal infusion rate, the target blood pressure, and the duration of SSFR. It may also be influenced by the depth of $\mathrm{TH}$ and its duration, and it is even more challenging to optimize the duration of TH and SSFR because they may influence each other. AFR should be carried out following SSFR until the desired resuscitation endpoints are achieved. The whole resuscitation management of traumatic uncontrolled HS from injury to hospital care, therefore, would be changed to LFR (before hemostasis), SSFR (after initial hemostasis), and AFR (after SSFR). The goal of this resuscitation strategy is to provide patients with best trauma care, which is called trauma-adapted resuscitation (TAR). This resuscitation strategy combined with $\mathrm{TH}$ may become the primary treatment for traumatic uncontrolled HS.

\section{Life expectancy oriented protection}

Current laboratory studies on traumatic HS have rarely considered the influence of cell survival on life expectancy of the body. Theoretically, however, salvage of special cells, like cardiomyocytes and neurons, is vital for improving disability-adjusted life years (DALYs), which implies prolonged life expectancy. It has long been believed that adult mammalian brain and spinal cord do not regenerate following injury, although some recent studies have shown neurogenesis in several regions of the central nervous system [40]. More well known is that about 20 million cardiomyocytes per year are lost due to ageing in the human left ventricle (two to four billion in total) [41]. In the case of cardiomyocyte and neuron death from trauma, these cells would not be substituted by homogeneous cells in adult human but lead to the formation of scar tissue, resulting in decreased or disordered organ function. Insult from severe traumatic HS may accelerate death of critical cells and shorten life span, while this process could be reversed by $\mathrm{TH}$. 


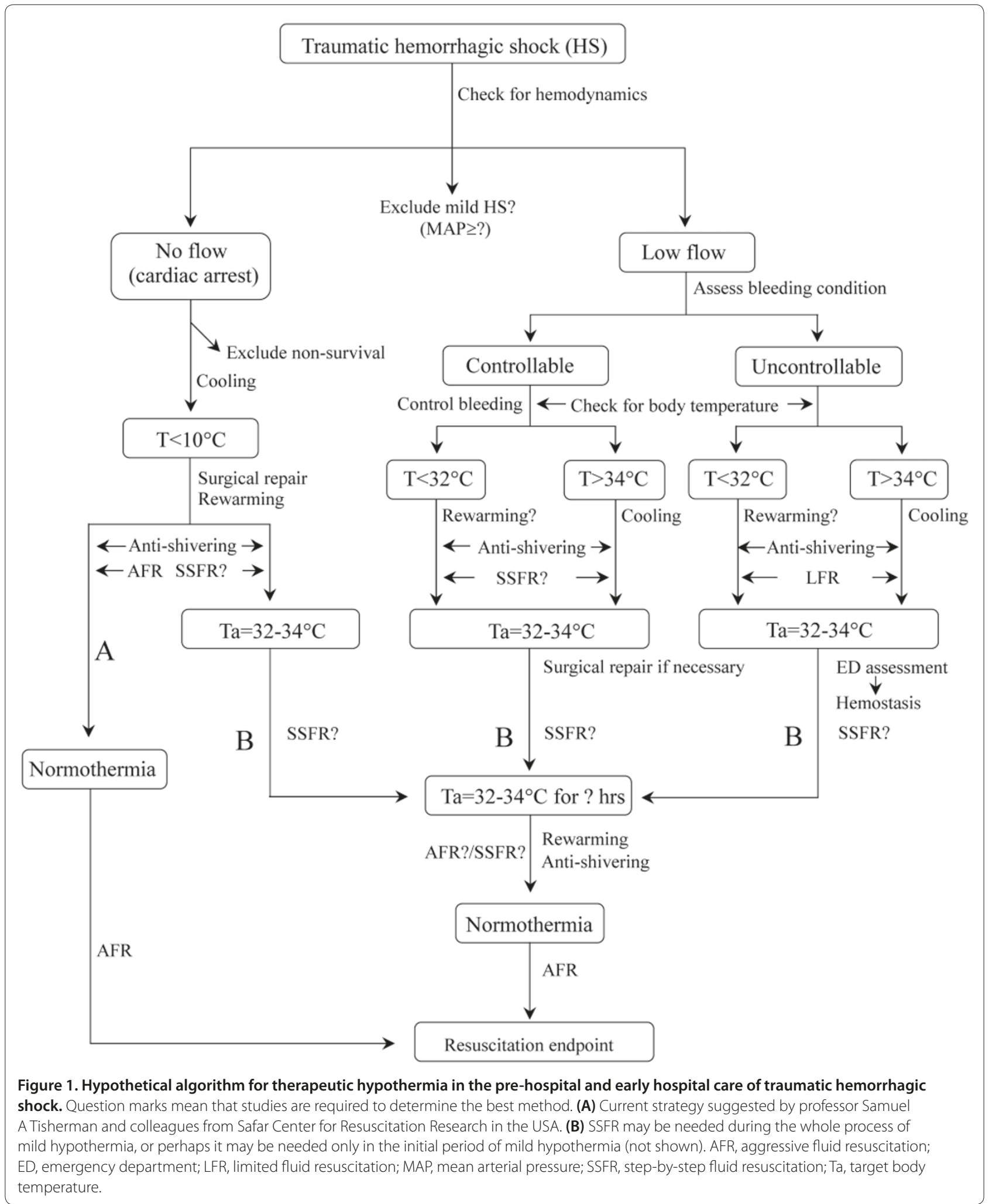

Barriers to translating basic evidence into practice Although $\mathrm{TH}$ has been suggested to assist patients recovering from traumatic $\mathrm{HS}$, its translation from basic research to the clinic confronts a spectrum of barriers. The major issues involve the induction and maintenance phase of $\mathrm{TH}$. Clinically, for patients with atraumatic 
cardiac arrest, mild TH could be feasible in the prehospital phase by intravenously infusing a large volume of $4^{\circ} \mathrm{C}$ fluids. However, with special emphasis on LFR for bleeding trauma, intravenous cooling alone may increase bleeding but could be efficient when LFR is combined with surface cooling. Nonetheless, surface cooling is relatively less effective for reaching the desired cooling state. For EPR treatment, the induction of deep-toprofound hypothermia through aortic perfusion in the pre-hospital phase could also be feasible because saline could remain chilled in ambulances for 24 hours using ice packs and coolers without significant cost [42]. However, pre-hospital deep-to-profound $\mathrm{TH}$ would be carried out without temperature-monitoring devices because of limited electricity, and the EPR treatment may suffer from logistic problems and availability of medical specialists. In addition, this is usually considered expensive and labor-intensive work. Thus, the safety and feasibility of pre-hospital EPR treatment should be carefully evaluated. Meanwhile, potential side effects during perioperative mild or deep-to-profound $\mathrm{TH}$ should be explored, as perioperative unintentional hypothermia is thought to be detrimental. On the other hand, the maintenance of target $\mathrm{TH}$ in uncontrolled $\mathrm{HS}$ is a serious issue. Surface rewarming methods may not be effective for precisely controlling target body temperature before hemostasis. In fact, it is even more challenging when SSFR and AFR are initiated because infusion of various fluids may rapidly decrease the body temperature without sensitive rewarming measures. Therefore, after hemostasis, commercialized endovascular cooling devices that can both induce and control hypothermia should be used [43].

\section{Algorithm development for use in therapeutic hypothermia}

Trauma patients are different with regard to injury type and severity, the extent of shock, volume of lost blood, underlying disease, and age. Thus, it is difficult to develop a common strategy for use of TH. Nonetheless, a clear framework is required. Herein, we have developed our initial hypothetical algorithm for use of $\mathrm{TH}$ in the treatment of traumatic HS based on existing data and the theoretical discussion above (Figure 1). This algorithm has revealed that a) patients with different blood flow states and bleeding conditions will be treated differently; b) mild HS may be excluded because those patients may not benefit from TH treatment; c) patients in HS would be maintained at 32 to $34^{\circ} \mathrm{C}$ regardless of the level of body temperature; d) for hemodynamically stable patients, the initial resuscitation strategy would be SSFR rather than AFR after brief hemostasis; and e) for exsanguination cardiac arrest patients, the EPR treatment may be updated by step rewarming and TAR - in other words, performing mild $\mathrm{TH}$ after deep-to-profound $\mathrm{TH}$ and performing SSFR after surgical control of bleeding. A couple of key questions remain to be answered before development of a final algorithm. Will there be indicators determining the duration of hypothermia treatment? And what is the best method of resuscitation when rewarming patients from mild hypothermia to normothermia, AFR or SSFR?

\section{Conclusion}

Hemorrhage following trauma continues to be a threat for both civilian and military patients. Much attention has been paid to the possibility of improving the outcome of traumatic HS by TH treatment. Correspondingly, it has been proposed that $\mathrm{TH}$ would not exacerbate the development of the trauma 'triad of death'. Even spontaneous hypothermia would not be viewed as completely harmful. The novel concept of TAR, including LFR, SSFR, and AFR, may change current resuscitation strategies in the treatment of uncontrolled traumatic HS. $\mathrm{TH}$ treatment in combination with TAR may provide patients with better trauma care. A specific algorithm for the use of mild or deep-to-profound TH will be useful for trauma surgeons and emergency, anesthesiology and critical care physicians. However, the mechanisms of action, the safety, and pre-hospital technical and logistic problems of $\mathrm{TH}$ still need to be determined. In addition, it is noteworthy that our algorithm is developed from basic evidence without clinical verification. All the hypotheses are raised for further laboratory study and prospective clinical trials. For more suggestions and insights, please refer to a recent meeting abstract [10].

\section{Abbreviations}

AFR, aggressive fluid resuscitation; EPR, emergency preservation and resuscitation; HS, hemorrhagic shock; LFR, limited fluid resuscitation; SSFR, step-by-step fluid resuscitation; TAR, trauma-adapted resuscitation; TH, therapeutic hypothermia.

\section{Competing interests}

The authors declare that they have no competing interests.

\section{Acknowledgements}

This work was supported by the Special Research Foundation of Public Health Industry, Ministry of Health, PR China (201002014).

\section{Published: 24 May 2013}

\section{References}

1. Soreide K, Kruger AJ, Vardal AL, Ellingsen CL, Soreide E, Lossius HM: Epidemiology and contemporary patterns of trauma deaths: changing place, similar pace, older face. World J Surg 2007, 31:2092-2103.

2. Champion HR, Bellamy RF, Roberts CP, Leppaniemi A: A profile of combat injury. J Trauma 2003, 54(5 Suppl):S13-19.

3. Cothren CC, Moore EE, Hedegaard HB, Meng K: Epidemiology of urban trauma deaths: a comprehensive reassessment 10 years later. World J Surg 2007, 31:1507-1511.

4. Kauvar DS, Lefering R, Wade CE: Impact of hemorrhage on trauma outcome: an overview of epidemiology, clinical presentations, and therapeutic considerations. J Trauma 2006, 60(6 Suppl):S3-11.

5. Sauaia A, Moore FA, Moore EE, Moser KS, Brennan R, Read RA, Pons PT: 
Epidemiology of trauma deaths: a reassessment. J Trauma 1995, 38:185-193.

6. Anderson ID, Woodford M, de Dombal FT, Irving M: Retrospective study of 1000 deaths from injury in England and Wales. Br Med J (Clin Res Ed) 1988, 296:1305-1308.

7. Blackbourne LH, Baer DG, Cestero RF, Inaba K, Rasmussen TE: Exsanguination shock: the next frontier in prevention of battlefield mortality. J Trauma 2011, 71(1 Suppl):S1-3.

8. Maybhate A, Hu C, Bazley FA, Yu Q, Thakor NV, Kerr CL, All AH: Potential longterm benefits of acute hypothermia after spinal cord injury: assessments with somatosensory-evoked potentials. Crit Care Med 2012, 40:573-579.

9. Varon J, Acosta P: Therapeutic hypothermia: past, present, and future. Chest 2008, 133:1267-1274.

10. Alam HB, Pusateri AE, Kindzelski A, Egan D, Hoots K, Andrews MT, Rhee P, Tisherman S, Mann K, Vostal J, Kochanek PM, Scalea T, Deal V, Sheppard F, Sopko G: Hypothermia and hemostasis in severe trauma: A new crossroads workshop report. J Trauma Acute Care Surg 2012, 73:809-817.

11. Fukudome EY, Alam HB: Hypothermia in multisystem trauma. Crit Care Med 2009, 37(7 Suppl):S265-272.

12. CliniclaTrials.gov: Emergency Preservation and Resuscitation (EPR) for Cardiac Arrest From Trauma (EPR-CAT) [http://clinicaltrials.gov/ct2/show/ NCT01042015?term=EPR\&rank=2]

13. Mikhail J: The trauma triad of death: hypothermia, acidosis, and coagulopathy. AACN Clin Issues 1999, 10:85-94

14. Kheirbek T, Kochanek AR, Alam HB: Hypothermia in bleeding trauma: a friend or a foe? Scand J Trauma Resusc Emerg Med 2009, 17:65.

15. Tisherman SA: Hypothermia and injury. Curr Opin Crit Care 2004, 10:512-519.

16. Alam HB: Translational barriers and opportunities for emergency preservation and resuscitation in severe injuries. Br J Surg 2012. 99 (Suppl 1):29-39.

17. Tisherman SA: Suspended animation for resuscitation from exsanguinating hemorrhage. Crit Care Med 2004, 32(2 Suppl):S46-50.

18. Kim K, Kim W, Rhee JE, Jo YH, Lee JH, Kim KS, Kwon WY, Suh GJ, Lee CC, Singer $\mathrm{AJ}$ : Induced hypothermia attenuates the acute lung injury in hemorrhagic shock. J Trauma 2010, 68:373-381.

19. Shuja F, Tabbara M, Li Y, Liu B, Butt MU, Velmahos GC, DeMoya M, Alam HB: Profound hypothermia decreases cardiac apoptosis through Akt survival pathway. J Am Coll Surg 2009, 209:89-99.

20. Childs EW, Udobi KF, Hunter FA: Hypothermia reduces microvascular permeability and reactive oxygen species expression after hemorrhagic shock. J Trauma 2005, 58:271-277.

21. Tanaka T, Wakamatsu T, Daijo H, Oda S, Kai S, Adachi T, Kizaka-Kondoh S, Fukuda K, Hirota K: Persisting mild hypothermia suppresses hypoxiainducible factor-1alpha protein synthesis and hypoxia-inducible factor-1mediated gene expression. Am J Physiol Regul Integr Comp Physiol 2010, 298:R661-671.

22. Li J, Beiser DG, Wang H, Das A, Berdyshev E, Leff AR, Stern SA, Vanden HoekTL: Therapeutic hypothermia cardioprotection in murine hemorrhagic shock/ resuscitation differentially affects p38alpha/p38gamma, Akt, and HspB1. J Trauma 2011, 71:1262-1270

23. Chen Z, Chen H, Rhee P, Koustova E, Ayuste EC, Honma K, Nadel A, Alam HB: Induction of profound hypothermia modulates the immune/ inflammatory response in a swine model of lethal hemorrhage. Resuscitation 2005, 66:209-216.

24. Meyer DM, Horton JW: Effect of moderate hypothermia in the treatment of canine hemorrhagic shock. Ann Surg 1988, 207:462-469.

25. Polderman $\mathrm{KH}$, Herold I: Therapeutic hypothermia and controlled normothermia in the intensive care unit: practical considerations, side effects, and cooling methods. Crit Care Med 2009, 37:1 101-1120.

26. Ireland S, Endacott R, Cameron P, Fitzgerald M, Paul E: The incidence and significance of accidental hypothermia in major trauma--a prospective observational study. Resuscitation 2011, 82:300-306.
27. Beilman GJ, Blondet JJ, Nelson TR, Nathens AB, Moore FA, Rhee P, Puyana JC, Moore EE, Cohn SM: Early hypothermia in severely injured trauma patients is a significant risk factor for multiple organ dysfunction syndrome but not mortality. Ann Surg 2009, 249:845-850.

28. Camp-Rogers T, Murphy G, Dean A, Gunnerson K, Rossler D, Kurz MC: Therapeutic hypothermia after profound accidental hypothermia and cardiac arrest. Am J Emerg Med 2012, 30:387 e385-387.

29. Martin RS, Kilgo PD, Miller PR, Hoth JJ, Meredith JW, Chang MC: Injuryassociated hypothermia: an analysis of the 2004 National Trauma Data Bank. Shock 2005, 24:114-118.

30. Mommsen P. Andruszkow H, Fromke C, Zeckey C, Wagner U, van Griensven $M$, Frink M, Krettek C, Hildebrand F: Effects of accidental hypothermia on posttraumatic complications and outcome in multiple trauma patients. Injury 2013, 44:86-90

31. Hess JR, Brohi K, Dutton RP, Hauser CJ, Holcomb JB, Kluger Y, Mackway-Jones K, Parr MJ, Rizoli SB, Yukioka T, Hoyt DB, Bouillon B: The coagulopathy of trauma: a review of mechanisms. J Trauma 2008, 65:748-754.

32. Frith D, Davenport R, Brohi K: Acute traumatic coagulopathy. Curr Opin Anaesthesiol 2012, 25:229-234

33. Holcomb JB, Jenkins D, Rhee P, Johannigman J, Mahoney P, Mehta S, Cox ED, Gehrke MJ, Beilman GJ, Schreiber M, Flaherty SF, Grathwohl KW, Spinella PC, Perkins JG, Beekley AC, McMullin NR, Park MS, Gonzalez EA, Wade CE, Dubick MA., Schwab CW, Moore FA, Champion HR, Hoyt DB, Hess JR: Damage control resuscitation: directly addressing the early coagulopathy of trauma. J Trauma 2007, 62:307-310.

34. Gruen RL, Brohi K, Schreiber M, Balogh ZJ, Pitt V, Narayan M, Maier RV: Haemorrhage control in severely injured patients. Lancet 2012, 380:1099-1108.

35. Husain FA, Martin MJ, Mullenix PS, Steele SR, Elliott DC: Serum lactate and base deficit as predictors of mortality and morbidity. Am J Surg 2003, 185:485-491

36. Cotton BA, Guy JS, Morris JA Jr, Abumrad NN: The cellular, metabolic, and systemic consequences of aggressive fluid resuscitation strategies. Shock 2006, 26:115-121.

37. Li T, Zhu Y, Fang Y, Liu L: Determination of the optimal mean arterial pressure for postbleeding resuscitation after hemorrhagic shock in rats. Anesthesiology 2012, 116:103-112.

38. Douzinas EE: Hemorrhagic shock resuscitation: a critical issue on the development of posttraumatic multiple organ failure. Crit Care Med 2012, 40:1348-1349.

39. Douzinas EE, Betrosian A, Giamarellos-Bourboulis EJ, Tasoulis MK, Prigouris P, Livaditi O, Andrianakis I, Goutas N, Vlachodimitropoulos D, Pelekanou A, Pelekanou A, Villiotou V, Legakis I, Chrousos GP: Hypoxemic resuscitation from hemorrhagic shock prevents lung injury and attenuates oxidative response and IL-8 overexpression. Free Radic Biol Med 2011, 50:245-253.

40. Gould E: How widespread is adult neurogenesis in mammals? Nat Rev Neurosci2007 8:481-488.

41. Laflamme MA, Murry CE: Heart regeneration. Nature 2011, 473:326-335.

42. Isenberg $D L$, Pasirstein $M J$ : A simple method of maintaining chilled saline in the prehospital setting. Am J Emerg Med 2012, 30:1385-1388

43. Pichon N, Amiel JB, Francois B, Dugard A, Etchecopar C, Vignon P: Efficacy of and tolerance to mild induced hypothermia after out-of-hospital cardiac arrest using an endovascular cooling system. Crit Care 2007, 11:R71 .

doi:10.1186/cc12559

Cite this article as: Jiang S, et al.: Potential role of therapeutic hypothermia

in the salvage of traumatic hemorrhagic shock. Critical Care 2013, 17:318. 
Traumatic hemorrhagic shock (HS)

Check for hemodynamics

No flow

(cardiac arrest)

Exclude mild HS?

(MAP $\geq$ ?)
Low flow

Assess bleeding condition

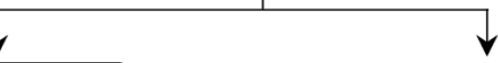

Cooling

$\mathrm{T}<10^{\circ} \mathrm{C}$

Surgical repair

Rewarming

$\leftarrow$ Anti-shivering $\rightarrow$

$\leftarrow$ AFR SSFR?

A

$\mathrm{Ta}=32-34^{\circ} \mathrm{C}$

Normothermia

B

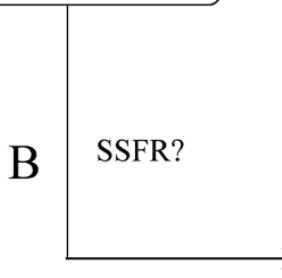

Controllable

\section{Control bleeding}

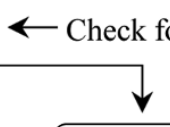

$\mathrm{T}<32^{\circ} \mathrm{C}$

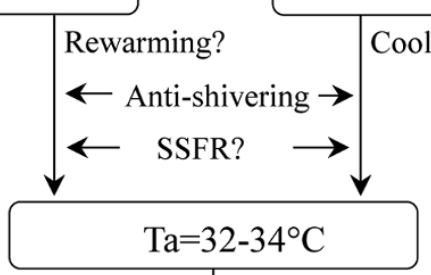

Surgical repair if necessary

Uncontrollable

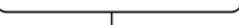

AFR

Normothermia

AFR 